\title{
Association between lifestyle factors and decreased kidney function in older adults: a community-based cross-sectional analysis of the Taipei City elderly health examination database
}

\author{
Horng-Jinh Chang ${ }^{1}$, Kuan-Reng Lin ${ }^{1 *} \mathbb{D}$, Meng-Te Lin² and Junn-Liang Chang ${ }^{2,3^{*}}$
}

\begin{abstract}
Background: Impaired kidney function is the hallmark of chronic kidney disease (CKD), and is associated with increased risk of all-cause mortality in the elderly. In the present cross-sectional population-based study, we aimed to evaluate the associations between lifestyle factors (exercise habit, alcohol consumption, smoking history, and betel nut chewing) and decreased kidney function.

Methods: The data from the Taipei City Elderly Health Examination Database (2006 to 2012) were extracted. Associations between risk factors and reduced estimated Glomerular filtration rate (eGFR) were evaluated by regression and stratification analyses.

Results: A total of 297,603 participants were included in the final analysis, and $29.7 \%$ of them had reduced eGFR. Smoking was significantly associated with an elevated risk of reduced eGFR. While, physical exercise conferred to a significantly decreased adjusted odds ratio (aOR) in reduced eGFR (regular exercise, aOR $=0.79$; occasional exercise, $\mathrm{aOR}=0.87$ ). Furthermore, the protective effect of exercise habit against reduced eGFR was not affected by comorbid conditions, such as hypertension, diabetes, obesity, and cardiovascular disease.
\end{abstract}

Conclusions: Engaging in physical exercise was beneficially associated with reduced eGFR in older individuals. Longitudinal or prospective studies are warranted for confirmation and extrapolation of the current findings.

Keywords: Kidney function, Glomerular filtration rate, Exercise, Comorbidity, Risk factor

\section{Background}

Chronic kidney disease (CKD) is a debilitating disease that affects around $11-13 \%$ of the global population and is estimated prevalent in 1.5 million individuals in

\footnotetext{
* Correspondence: kittylintku@gms.tku.edu.tw; junn9liang@yahoo.com.tw 'Department of Management Sciences, Tamkang University, No.151,

Yingzhuan Rd., Tamsui Dist, New Taipei City 25137, Taiwan, Republic of China 2 Department of Pathology \& Laboratory Medicine, Taoyuan Armed Forces General Hospital, No.168, Chung-Shing Rd., Long-Tang District, Taoyuan City 325, Taiwan, Republic of China

Full list of author information is available at the end of the article
}

Taiwan $[1,2]$. A Korean National Health and Nutrition Evaluation Survey study indicated that the CKD prevalence rates were 3.5 and $2.4 \%$ in male and female adults, respectively, in 2013 in South Korea [3]. In addition, among European general populations, the CKD prevalence varied from $3.31 \%$ in Norway to $17.3 \%$ in northeast Germany [4]. Furthermore, a systematic review and meta-analysis of observational studies concluded that in general populations, the CKD prevalence rates were 3.5, 3.9, 7.6, 0.4, and 0.1\% for CKD Stage 1, Stage 2, Stage 3,

(c) The Author(s). 2020 Open Access This article is licensed under a Creative Commons Attribution 4.0 International License, which permits use, sharing, adaptation, distribution and reproduction in any medium or format, as long as you give appropriate credit to the original author(s) and the source, provide a link to the Creative Commons licence, and indicate if changes were made. The images or other third party material in this article are included in the article's Creative Commons licence, unless indicated otherwise in a credit line to the material. If material is not included in the article's Creative Commons licence and your intended use is not permitted by statutory regulation or exceeds the permitted use, you will need to obtain permission directly from the copyright holder. To view a copy of this licence, visit http://creativecommons.org/licenses/by/4.0/. The Creative Commons Public Domain Dedication waiver (http://creativecommons.org/publicdomain/zero/1.0/) applies to the data made available in this article, unless otherwise stated in a credit line to the data. 
Stage 4, and Stage 5, respectively [1]. However, according to a large-scale National Health and Nutrition Evaluation Survey study in the US, the CKD prevalence is $46.8 \%$ in elderly people aged over 70 years, which is much higher than that in younger individuals [5]. CKD is diagnosed by the presence of abnormalities in estimated glomerular filtration rate (eGFR) and markers of kidney damage that include albuminuria and hematuria for more than 3 months [6]. A person with eGFR $<60$ $\mathrm{ml} / \mathrm{min} / 1.73 \mathrm{~m}^{2}$ is said to have impaired kidney function. It has been shown that lower eGFR and albuminuria are associated with higher prevalence and incidence of kidney failure, cardiovascular disease (CVD), CVDassociated mortality, and all-cause mortality in the elderly [7-11]. Alternatively, CVD itself and its risk factors, including diabetes, obesity and hypertension, are associated with the incidence and progression of CKD [12].

Physical activity and exercise have been shown to improve the quality of life and reduce the risk of dyslipidemia and CVD in the elderly [13-15]. A good physical performance is associated with reduction in all-cause mortality among older individuals with CKD [16]. Nevertheless, the benefits of physical exercise in preventing CKD or impaired kidney function are under debate. A cross-sectional study showed that higher levels of physical activity and lower levels of sedentary behavior were associated with reduced risk for lower eGFR [17]. However, the Framingham Heart Study found no significant association between physical activity and incident eGFR $<60$ or rapid eGFR decline [18].

As the world population is aging, it is estimated that over 1.6 billion people worldwide will be 65 years and older by 2050, projecting an increasing burden on the health care system and society [19]. Given the unfavorable outcomes and increased prevalence of comorbidities in older people with CKD, primary care and preventive lifestyle measures are both important aspects in maintaining kidney health in these individuals [20]. Herein, we hypothesized that lifestyle factors may affect the risk for reduced eGFR in adults aged 65 or over. To examine this hypothesis, we conducted this cross-sectional population-based study to evaluate the associations between reduced eGFR and various lifestyle factors, including exercise habit, alcohol consumption, smoking history, and betel nut chewing, in a community-dwelling elderly population in Taipei, Taiwan.

\section{Methods}

\section{Data source and study population}

The present study analyzed the data derived from the Taipei City Elderly Health Examination Database (2006 to 2012), which collected health examination data from recruited community-dwelling Taipei citizens aged 65 years or older. The Taipei City Elderly Health
Examination Database is sponsored by the Department of Health, Taipei City Government, and has been used for the purpose of research as previously described [13, 21-23]. In the present cross-sectional study, participants who had missing values for age, gender, or the serum creatinine level were excluded, because all these variables were required for the calculation of eGFR based on the Chronic Kidney Disease Epidemiology Collaboration (CKD-EPI) formula [24]. In addition, participants who had renal cancer, kidney disease, nephrectomy, kidney transplantation, and renal failure were also excluded.

\section{Ethical considerations}

The present cross-sectional population-based study was approved by the institutional review board of the Taipei City Hospital (TCHIRB-10514118-W), and informed consent was waived due to the retrospective nature of this study. All participants of the Taipei City Elderly Health Examination Database provided written informed consent at the time of health examination, authorizing the Taipei City Government access to their data for research purposes. All personal identifying information had been delinked in the Taipei City Elderly Health Examination Database.

\section{Data extraction}

The participants' cross-sectional demographic and socioeconomic characteristics, lifestyle factors, blood and urine variables, and comorbidity were extracted from the Taipei City Elderly Health Examination Database. The demographic and socioeconomic characteristics included age, gender, marital status, body mass index (BMI), highest education degree, and income level. Lifestyle factors consisted of exercise habit, alcohol consumption, smoking history, and betel nut chewing. Blood and urine variables were the levels of fasting glucose, total cholesterol, triglyceride, high density lipoprotein (HDL), urine acid, blood urea nitrogen (BUN), creatinine, and proteinuria. Finally, the comorbidities such as hypertension, diabetes mellitus, dyslipidemia, hyperuricemia, urinary tract stones, CVD, and cancer were analyzed.

\section{Statistical analysis}

Descriptive statistics of the participants' demographic characteristics and blood and urine variables were presented as number (n) and percentage (\%) or mean \pm standard deviation (SD). Differences in categorical variables were tested by Pearson's chi-square test, and those in numerical variables by two-sample t-test. Logistic regression analyses were performed to identify the risk factors associated with decreased kidney function (defined as eGFR $<60 \mathrm{ml} / \mathrm{min} / 1.73 \mathrm{~m}^{2}$ in the present study). Multivariate logistic regression analysis was performed in the total population after adjusting for all variables 
that were significant in the univariate regression model. Furthermore, participants were further stratified based on gender, hypertension, diabetes, obesity, and CVD to assess the possible confounding effect of gender, hypertension, diabetes, obesity, or CVD on association between exercise habit and reduced kidney function. In the stratified analysis, multivariate model was adjusted for other significant variables identified in the univariate regression model in the total population. All statistical analyses were two-sided and a $p$ value $<0.05$ was considered statistically significant. All statistical analyses were performed using SAS software version 9.4 (SAS Institute Inc., Cary, NC, USA).

\section{Results}

The present cross-sectional study accessed the data derived from 346,084 community-dwelling individuals aged 65 and over who participated in the Taipei City Elderly Health Examination program between 2006 and 2012. Altogether 41,778 participants who missing data for age, gender, or the serum creatinine level were excluded. In addition, 6703 participants who had history of renal cancer, kidney disease, nephrectomy, kidney transplantation, or renal failure were also excluded. As a result, a total of 297,603 participants were included in the final analysis (Fig. 1).
Demographic, socioeconomic, lifestyle factors, blood and urine variables, and comorbidities of the total population are presented in Table 1 . The mean age of all participants was 75 years, and $51.5 \%$ of them were male. The majority of participants were married/cohabiting (73.8\%), and $46.1 \%$ of participants had normal BMI. Regarding socioeconomic characteristics, 49.8\% had high school diploma or higher, and 95.7\% were financially better than poor. Most participants had regular exercise habit (49.6\%), no drinking habit (97.6\%), no current smoking habit (93.0\%), and no betel-nut chewing habit $(99.1 \%)$. In the present cohort, dyslipidemia (66.3\%), hypertension (43.5\%), CVD (20.8\%), hyperuricemia/gout (19.9\%), and diabetes (17.5\%) were present as comorbidities, and $8.1 \%$ of participants had proteinuria (Table 1).

Included participants were then divided into two groups based on eGFR variables: the reduced eGFR group (participants with eGFR $<60 \mathrm{ml} / \mathrm{min} / 1.73 \mathrm{~m}^{2}$; $n=88,219,29.7 \%$ ) and the normal eGFR group (participants with eGFR $\geq 60 \mathrm{ml} / \mathrm{min} / 1.73 \mathrm{~m}^{2} ; n=209,384$, 70.3\%). Demographic, socioeconomic, lifestyle factors, blood and urine variables, and comorbidities of the two groups are shown in Table 2. Almost all demographic, socioeconomic, and lifestyle factors including physical exercise were significantly different between

$$
\begin{aligned}
& \text { Community-dwelling senior citizens participating in } \\
& \text { the Taipei City Elderly Health Examination program } \\
& \text { between } 2006 \text { and } 2012 \\
& (\mathrm{n}=346,084)
\end{aligned}
$$

\section{Exclusion criteria}

1. Participants with missing data for age, gender, or the serum creatinine level $(n=41,778)$

2. Participants who had history of renal cancer, kidney disease, nephrectomy, kidney transplantation, or renal failure $(\mathrm{n}=6,703)$

Study population

$(\mathrm{n}=297,603)$

Fig. 1 Flow chart of the study population 
Table 1 Demographic, socioeconomic, and lifestyle factors of the total study population

\begin{tabular}{|c|c|}
\hline Variables & Total $^{a}(n=297$ \\
\hline \multicolumn{2}{|l|}{ Demographics } \\
\hline Age & $75.0 \pm 6.7$ \\
\hline \multicolumn{2}{|l|}{ Gender } \\
\hline Female & $144,260(48.5)$ \\
\hline Male & $153,343(51.5)$ \\
\hline \multicolumn{2}{|l|}{ Marital status } \\
\hline Married/cohabiting & $219,615(73.8)$ \\
\hline Widowed/divorced/separated & $62,041(20.9)$ \\
\hline Never married & $14,277(4.8)$ \\
\hline Missing value & $1670(0.5)$ \\
\hline \multicolumn{2}{|l|}{$\mathrm{BMI}^{\mathrm{b}}$} \\
\hline Underweight & $11,484(3.9)$ \\
\hline Normal & $137,246(46.1)$ \\
\hline Overweight & $93,162(31.3)$ \\
\hline Obese & $53,068(17.8)$ \\
\hline Missing value & $2643(0.9)$ \\
\hline \multicolumn{2}{|l|}{ Socioeconomic factors } \\
\hline \multicolumn{2}{|l|}{ Educational attainment } \\
\hline With high school diploma or higher degree & $148,298(49.8)$ \\
\hline Without high school diploma & $141,261(47.5)$ \\
\hline Missing value & $8044(2.7)$ \\
\hline \multicolumn{2}{|l|}{ Income level } \\
\hline Not poor & $284,808(95.7)$ \\
\hline Poor & $12,795(4.3)$ \\
\hline \multicolumn{2}{|l|}{ Lifestyle factors } \\
\hline \multicolumn{2}{|l|}{ Exercise habit } \\
\hline No & $33,021(11.1)$ \\
\hline Occasional & $93,125(31.3)$ \\
\hline Regular & $147,554(49.6)$ \\
\hline Missing value & $23,903(8.0)$ \\
\hline \multicolumn{2}{|l|}{ Alcohol drinking } \\
\hline No & $290,524(97.6)$ \\
\hline Yes & $6052(2.0)$ \\
\hline Missing value & $1027(0.4)$ \\
\hline \multicolumn{2}{|l|}{ Current smoking } \\
\hline No & $276,610(93.0)$ \\
\hline Yes & $20,047(6.7)$ \\
\hline Missing value & $946(0.3)$ \\
\hline \multicolumn{2}{|l|}{ Betel nut chewing } \\
\hline No & 294,898 (99.1) \\
\hline Yes & $1041(0.4)$ \\
\hline Missing value & $1664(0.5)$ \\
\hline
\end{tabular}

Blood and urine variables
Table 1 Demographic, socioeconomic, and lifestyle factors of the total study population (Continued)

\begin{tabular}{ll}
\hline Variables & Total $^{\mathrm{a}}(n=297,603)$ \\
\hline Fasting glucose & $104.7 \pm 24.4$ \\
Total cholesterol & $194.4 \pm 35.2$ \\
Triglyceride & $120.7 \pm 72.9$ \\
HDL & $54.6 \pm 14.9$ \\
Uric acid & $5.9 \pm 1.6$ \\
BUN & $17.6 \pm 5.8$ \\
Creatinine & $1.0 \pm 0.4$ \\
Proteinuria & $24,165(8.1)$ \\
Comorbidity & \\
Hypertension & $129,492(43.5)$ \\
Diabetes & $52,186(17.5)$ \\
Dyslipidemia & $197,373(66.3)$ \\
Hyperuricemia/gout & $59,201(19.9)$ \\
Urinary tract stones & $6464(2.2)$ \\
Cardiovascular disease & $61,895(20.8)$ \\
Cancer & $1558(0.5)$
\end{tabular}

${ }^{a}$ Data of the total study population were presented as mean \pm standard deviation or number of participants (\% of total cohort)

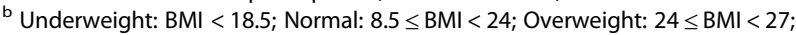

Obese: $\mathrm{BMI} \geq 27$

' Some participants had multiple comorbidities

Abbreviation: $B M I$ body mass index; $B U N$ blood urea nitrogen; $H D L$ high density lipoprotein

two eGFR groups, except for alcohol drinking ( $p=$ 0.1268) (Table 2). The reduced eGFR group had an older mean age and were more often male than the normal eGFR group. Compared with participants with normal eGFR, participants with decreased kidney function had slightly different fasting glucose, total cholesterol, triglyceride, and HDL levels, but these differences were small and of little clinical significance. In contrast, the differences in uric acid, BUN, creatinine, and percentage of participants with proteinuria were more pronounced in the two groups and were statistically significant. For comorbidities, significantly higher percentages of hypertension (53.9\%), diabetes $(22.5 \%)$, dyslipidemia (68.5\%), hyperuricemia/gout (36.5\%), and CVD (27.3\%) cases were observed in the reduced eGFR group compared to those of the normal eGFR group (Table 2).

The associations between decreased kidney function and demographic and socioeconomic characteristics, lifestyle factors, proteinuria, and comorbidity in the total study population were evaluated using logistic regression analyses (Table 3 ). The variables that were significant in univariate logistic regression analysis were included in the multivariate logistic regression model. The results of multivariate analysis revealed that participants who had either regular or occasional exercise habit consistently 
Table 2 Demographic, socioeconomic, and lifestyle factors of participants with decreased or normal eGFR

Variables
Demographics
Age
Gender
Female
Male
Marital status
Married/cohabiting
Widowed/divorced/separated
Never married
Missing value
BMl ${ }^{\text {a }}$
Underweight
Normal
Overweight
Obese
Missing value
Socioeconomic factors
Educational attainment
Wissing value
Income level poor
Nool diploma or higher degree
Wigh school diploma
Nith

\section{Lifestyle factors}

Exercise habit

No

Occasional

Regular

Missing value

Alcohol drinking

No

Yes

Missing value

Current smoking

No

Yes

Missing value

Betel nut chewing

No

Yes

Missing value eGFR $\left(\mathrm{ml} / \mathrm{min} / 1.73 \mathrm{~m}^{2}\right)$

Reduced (< 60)

$n=88,219$

Normal $(\geq 60)$

$p$-value $n=209,384$

$77.7 \pm 6.7$

$73.9 \pm 6.3$

$<0.001$

$<0.001$

35,035 (39.7)

$109,225(52.2)$

$53,184(60.3)$

100,159 (47.8)

$62,820(71.2)$

156,795 (74.9)

19,878 (22.5)

42,163 (20.1)

4812 (5.5)

$9465(4.5)$

$709(0.8)$

$961(0.5)$

2762 (3.1)

$8722(4.1)$

36,575 (41.5)

29,309 (33.2)

18,568 (21.1)

$1005(1.1)$

$100,671(48.1)$

63,853 (30.5)

$34,500(16.5)$

$1638(0.8)$

$<0.001$

$<0.001$

43,684 (49.5)

$104,614(50.0)$

41,575 (47.1)

99,686 (47.6)

2960 (3.4)

5084 (2.4)

$83,260(94.4)$

4959 (5.6)

201,548 (96.3)

7836 (3.7)

$<0.001$

$12,212(13.8)$

28,282 (32.1)

41,105 (46.6)

$6620(7.5)$

0.1268

$86,177(97.7)$

$1727(2.0)$

$315(0.3)$

20,809 (9.9)

64,843 (31.0)

106,449 (50.8)

17,283 (8.3)

204,347 (97.6)

4325 (2.1)

$712(0.3)$

$<0.001$

$81,224(92.1)$

195,386 (93.3)

6709 (7.6)

$286(0.3)$

13,338 (6.4)

$660(0.2)$

0.0236

207,428 (99.1)

$270(0.3)$

$771(0.4)$

$448(0.5)$ 
Table 2 Demographic, socioeconomic, and lifestyle factors of participants with decreased or normal eGFR (Continued)

\begin{tabular}{|c|c|c|c|}
\hline \multirow[t]{2}{*}{ Variables } & \multicolumn{3}{|c|}{ eGFR $\left(\mathrm{ml} / \mathrm{min} / 1.73 \mathrm{~m}^{2}\right)$} \\
\hline & $\begin{array}{l}\text { Reduced }(<60) \\
n=88,219\end{array}$ & $\begin{array}{l}\text { Normal }(\geq 60) \\
n=209,384\end{array}$ & $p$-value \\
\hline \multicolumn{4}{|l|}{ Blood and urine variables } \\
\hline Fasting glucose & $106.9 \pm 27.6$ & $103.8 \pm 22.9$ & $<0.001$ \\
\hline Total cholesterol & $191.9 \pm 36.1$ & $195.4 \pm 34.8$ & $<0.001$ \\
\hline Triglyceride & $130.8 \pm 77.9$ & $116.5 \pm 70.3$ & $<0.001$ \\
\hline $\mathrm{HDL}$ & $52.0 \pm 14.6$ & $55.7 \pm 14.8$ & $<0.001$ \\
\hline Uric acid & $6.6 \pm 1.7$ & $5.6 \pm 1.4$ & $<0.001$ \\
\hline BUN & $21.3 \pm 7.7$ & $16.0 \pm 3.9$ & $<0.001$ \\
\hline Creatinine & $1.3 \pm 0.5$ & $0.9 \pm 0.2$ & $<0.001$ \\
\hline Proteinuria & $13,758(15.6)$ & $10,407(5.0)$ & $<0.001$ \\
\hline \multicolumn{4}{|l|}{ Comorbidity } \\
\hline Hypertension & $47,553(53.9)$ & $81,939(39.1)$ & $<0.001$ \\
\hline Diabetes & $19,802(22.5)$ & $32,384(15.5)$ & $<0.001$ \\
\hline Dyslipidemia & $60,389(68.5)$ & $136,984(65.4)$ & $<0.001$ \\
\hline Hyperuricemia/gout & $32,220(36.5)$ & $26,981(12.9)$ & $<0.001$ \\
\hline Urinary tract stones & $1887(2.1)$ & $4577(2.2)$ & 0.4224 \\
\hline Cardiovascular disease & $24,101(27.3)$ & $37,794(18.1)$ & $<0.001$ \\
\hline Cancer & $478(0.5)$ & $1080(0.5)$ & 0.3688 \\
\hline
\end{tabular}

Data were presented as mean \pm standard deviation or number of participants (\% of variables)

${ }^{a}$ Underweight: $\mathrm{BMI}<18.5$; Normal: $8.5 \leq \mathrm{BMI}<24$; Overweight: $24 \leq \mathrm{BMI}<27$; Obese: $\mathrm{BMI} \geq 27$

Abbreviations: $B M I$ body mass index; $e G F R$ estimated glomerular filtration rate

showed significantly lower odds of having decreased kidney function than those who did not exercise (occasional: adjusted $\mathrm{OR}[\mathrm{aOR}]=0.87,95 \% \mathrm{CI}=0.84-0.90$; regular: $\mathrm{aOR}=0.79,95 \% \mathrm{CI}=0.77-0.81)$. Betel nutchewing was associated with lower risk of prevalent CKD in the univariate logistic regression model, but not after multivariate adjustment. In contrast, older age $(\mathrm{aOR}=1.08,95 \% \mathrm{CI}=1.08-1.09)$, and the status of never married $\quad(\mathrm{aOR}=1.10, \quad 95 \% \quad \mathrm{CI}=1.05-1.14)$, current smoker $(\mathrm{aOR}=1.15,95 \% \mathrm{CI}=1.11-1.19)$, proteinuria $(\mathrm{aOR}=2.56,95 \% \mathrm{CI}=2.49-2.64)$, dyslipidemia $(\mathrm{aOR}=$ $1.14,95 \% \mathrm{CI}=1.12-1.16)$, or hyperuricemia/gout $(\mathrm{aOR}=$ $3.39,95 \% \mathrm{CI}=3.32-3.46)$ were significantly associated with higher odds of having decreased kidney function. Furthermore, well-known indicators of CKD such as male gender $(\mathrm{aOR}=1.36,95 \% \mathrm{CI}=1.34-1.39)$, overweight/obesity (overweight: $\mathrm{aOR}=1.14,95 \% \mathrm{CI}=1.12$ 1.16; obese: $\mathrm{aOR}=1.16,95 \% \mathrm{CI}=1.13-1.19$; compared with reference group), hypertension ( $\mathrm{aOR}=1.33,95 \%$ $\mathrm{CI}=1.31-1.35)$, diabetes $(\mathrm{aOR}=1.26,95 \% \mathrm{CI}=1.23$ 1.29), and cardiovascular disease $(\mathrm{aOR}=1.25,95 \% \mathrm{CI}=$ 1.22-1.27) also showed higher odds of having decreased kidney function than those without the corresponding comorbidities (Table 3).

To examine whether gender, hypertension, diabetes, obesity, and cardiovascular disease affect the association between exercise habit and decreased kidney function, stratified analyses were performed (Table 4). The protective effect of exercise habit on the odds of decreased kidney function was observed in both male and female participants (female: $\mathrm{aOR}=0.80,95 \% \mathrm{CI}=0.77-0.83$; male: $\mathrm{aOR}=0.85,95 \% \mathrm{CI}=0.81-0.88)$, as determined by multivariate model after adjusting for age, marital status, current smoker, proteinuria, dyslipidemia and hyperuricemia/gout. The exercise habit was associated with significantly lower odds of decreased kidney function in participants with or without hypertension (hypertension: $\mathrm{aOR}=0.87,95 \% \mathrm{CI}=0.84-0.90$; without hypertension: $\mathrm{aOR}=0.84,95 \% \mathrm{CI}=0.81-0.87$ ), with or without diabetes (diabetes: $\mathrm{aOR}=0.83,95 \% \mathrm{CI}=0.78-0.87$; without diabetes: $\mathrm{aOR}=0.87,95 \% \mathrm{CI}=0.84-0.89$ ), with or without obesity (obesity: $\mathrm{aOR}=0.82,95 \% \mathrm{CI}=0.78-0.87$; without obesity: $\mathrm{aOR}=0.85,95 \% \mathrm{CI}=0.82-0.88)$, and with or without CVD (CVD: $\mathrm{aOR}=0.87,95 \% \mathrm{CI}=0.83-$ 0.92; without CVD: $a O R=0.85,95 \% \mathrm{CI}=0.83-0.88$ ) (Table 4).

\section{Discussions}

To the best of our knowledge, this is the largest crosssectional study involving 297,603 participants representing a generalized community-dwelling elderly population, which investigated the associations between 
Table 3 Associations between study variables and decreased kidney function (eGFR < 60) in the total study population

\begin{tabular}{|c|c|c|}
\hline & $\mathrm{OR}^{\mathrm{a}}(95 \% \mathrm{Cl})$ & $\mathrm{aOR}^{\mathrm{b}}(95 \% \mathrm{Cl})$ \\
\hline \multicolumn{3}{|l|}{ Demographics } \\
\hline Age & $1.09(1.09-1.09)^{*}$ & $1.08(1.08-1.09)^{*}$ \\
\hline \multicolumn{3}{|l|}{ Gender } \\
\hline Female & Ref. & Ref. \\
\hline Male & $1.66(1.63-1.68)^{*}$ & $1.36(1.34-1.39)^{*}$ \\
\hline \multicolumn{3}{|l|}{ Marital status } \\
\hline Married/cohabiting & Ref. & Ref. \\
\hline Widowed/divorced/separated & $1.18(1.15-1.20)^{*}$ & $1.02(0.99-1.04)$ \\
\hline Never married & $1.27(1.22-1.32)^{*}$ & $1.10(1.05-1.14)^{*}$ \\
\hline \multicolumn{3}{|l|}{$\mathrm{BMI}^{\mathrm{c}}$} \\
\hline Normal weight & Ref. & Ref. \\
\hline Underweight & $1.15(1.10-1.20)^{*}$ & $0.78(0.74-0.82)^{*}$ \\
\hline Overweight & $1.45(1.39-1.52)^{*}$ & $1.14(1.12-1.16)^{*}$ \\
\hline Obese & $1.70(1.62-1.78)^{*}$ & $1.16(1.13-1.19)^{*}$ \\
\hline
\end{tabular}

\section{Socioeconomic factors}

Educational attainment

With high school diploma or higher degree

Ref.

Without high school diploma school

$1.0(0.98-1.01)$

Income level

Not poor

Ref.

Ref.

Poor

$1.53(1.48-1.59)^{*}$

$1.03(0.98-1.07)$

\section{Lifestyle factors}

Exercise habit

No

Ref.

Ref.

Occasional

$0.74(0.72-0.76)^{*}$

$0.87(0.84-0.90)^{*}$

Regular

$0.66(0.64-0.67)^{*}$

$0.79(0.77-0.81)^{*}$

Alcohol drinking (vs. no)

$0.95(0.90-1.01)$

Current smoking (vs. no)

$1.21(1.17-1.25)^{*}$

$1.15(1.11-1.19)^{*}$

Betel nut chewing (vs. no)

\section{Blood and urine variables}

Proteinuria

Comorbidity

Hypertension

$1.82(1.79-1.85)^{*}$

$1.33(1.31-1.35)^{*}$

Diabetes

$1.58(1.55-1.61)^{*}$

$1.26(1.23-1.29)^{*}$

Dyslipidemia

$1.15(1.13-1.17)^{*}$

$1.14(1.12-1.16)^{*}$

Hyperuricemia/gout

$3.89(3.82-3.96)^{*}$

$3.39(3.32-3.46)^{*}$

Urinary tract stones

$0.98(0.93-1.03)$

Cardiovascular disease

$1.71(1.68-1.74)^{*}$

Cancer

$1.05(0.94-1.17)$

Note: Star $(*)$ indicates statistical significance $(p<0.05)$

a Univariate regression analysis

${ }^{\mathrm{b}}$ Multivariate regression analysis

c Underweight: $\mathrm{BMI}<$ 18.5; Normal: $8.5 \leq \mathrm{BMl}<24$; Overweight: $24<\mathrm{BMl}<27$; Obese: $\mathrm{BMI} \geq 27$

Abbreviation: $a O R$ adjusted $O R ; B M I$ body mass index; $C l$ confidence interval; $O R$ odds ration; Ref reference 
Table 4 Effects of gender, diabetes, hypertension, obesity and cardiovascular disease on the association between exercise habit and decreased kidney function, determined by stratified analyses

\begin{tabular}{|c|c|c|}
\hline \multirow[t]{2}{*}{ Stratification } & \multicolumn{2}{|c|}{ The association between exercise habit ${ }^{a}$ and decreased eGFR $(<60)$} \\
\hline & OR $(95 \% \mathrm{Cl})$ & $\mathrm{aOR}(95 \% \mathrm{Cl})$ \\
\hline \multicolumn{3}{|l|}{ Gender } \\
\hline Female & $0.60(0.58-0.62)^{*}$ & $0.80(0.77-0.83)^{*}$ \\
\hline Male & $0.70(0.68-0.73)^{*}$ & $0.85(0.81-0.88)^{*}$ \\
\hline \multicolumn{3}{|c|}{ Hypertension } \\
\hline Yes & $0.72(0.69-0.74)^{*}$ & $0.87(0.84-0.90)^{*}$ \\
\hline No & $0.68(0.66-0.71)^{*}$ & $0.84(0.81-0.87)^{*}$ \\
\hline \multicolumn{3}{|l|}{ Diabetes } \\
\hline Yes & $0.66(0.62-0.69)^{*}$ & $0.83(0.78-0.87)^{*}$ \\
\hline No & $0.72(0.70-0.74)^{*}$ & $0.87(0.84-0.89)^{*}$ \\
\hline \multicolumn{3}{|l|}{ Obesity $^{b}$} \\
\hline Yes & $0.73(0.69-0.77)^{*}$ & $0.82(0.78-0.87)^{*}$ \\
\hline No & $0.69(0.67-0.71)^{*}$ & $0.85(0.82-0.88)^{*}$ \\
\hline \multicolumn{3}{|c|}{ Cardiovascular disease } \\
\hline Yes & $0.70(0.67-0.74)^{*}$ & $0.87(0.83-0.92)^{*}$ \\
\hline No & $0.71(0.69-0.73)^{*}$ & $0.85(0.83-0.88)^{*}$ \\
\hline
\end{tabular}

Note: Star $(*)$ indicates statistical significance $(p<0.05)$

Multivariate model was adjusted for age, marital status, current smoker, proteinuria, dyslipidemia and hyperuricemia/gout

${ }^{a}$ Exercise habit was defined as with regular or occasional exercise

${ }^{b}$ Obesity was defined as $\mathrm{BMI} \geq 27$

Abbreviation: $a O R$ adjusted $\mathrm{OR}$; $\mathrm{Cl}$ confidence interval; eGFR estimated glomerular filtration rate; $O R$ odds ratio

lifestyle factors and reduced kidney function. The present study found that smoking was associated with increased odds of an impaired eGFR, while physical exercise was favorable for kidney function. Comorbid conditions including CVD, hypertension, obesity, and diabetes that are known to contribute to risk of developing CKD were also found associated with reduced eGFR in the present study. The reduced odds of having a decreased eGFR conferred by physical exercise habit in the presence of different comorbid conditions associated with CKD was further demonstrated by stratification analysis, where the benefit of physical exercise was consistently shown.

In line with the decreased odds of impaired kidney function associated with physical exercise observed by us, the Maastricht Study found higher physical activity beneficially associated with kidney function [25] This prospective observational population-based study recruited individuals aged between 40 and 75 years through public engagement, and further reported that sedentary behavior was associated with adverse kidney function [25]. Similarly, a cross-sectional study involving 1350 men with a mean age of 78.5 years showed that higher physical activity and a less sedentary behavior were associated with favorable kidney function [17], in which the level of physical activity and sedentary behavior were measured objectively over a 7-day period by wearable physical activity monitors. Although data concerning physical exercise habit and other lifestyle factors were collected via a selfadministered questionnaire in our study, the study by Parson et al. (2017) [17] strengthens our observation that both participant groups with occasional or regular exercise habit showed reduced odds of eGFR $<60$ compared with those who did not exercise. Furthermore, a meta-analysis research indicated that physical activity significantly reduces blood pressure in patients who are aged 50 and older and had chronic renal failure [26].

On the other hand, the Framingham Heart Study did not observe associations between baseline physical activity and incident impaired eGFR or rapid eGFR decline over a median follow-up of 6.6 years in older adults, who were offspring of the original Framingham cohort [18]. In addition, a pilot randomized controlled study tested the preventive benefits of exercise training on cardiovascular, vascular and renal health in adults with stage 3-4 CKD found that the 1-year exercise intervention did not slow the progression of kidney disease [27]. It should be noted that the two studies described above were prospective and longitudinal studies, and involved different age groups 
compared with the present study. Nevertheless, the disparity in findings stresses the need of further research to define the preventive role of active physical exercise in kidney health.

Supportive of our findings, previous studies reported smoking and betel-nut chewing as hazardous lifestyle factors with a negative impact on the risk of developing CKD $[28,29]$. In the present study, we further illustrate that these lifestyle habits are also unfavorable in people $>65$ years-old. On the other hand, despite the Korean National Health and Nutrition Examination Survey (KNHANES) showing negative correlation between alcohol drinking and renal dysfunction especially in men [30], this was not observed in the present study. Discrepancy results may be in part due to cultural differences in drinking, as around $76.5 \%$ of participants in the KNHANES study were moderate to heavy drinkers, in contrast to our cohort, where only $2 \%$ were alcohol drinkers. The other discrepancy was the age of participants, as we focused on elderly individuals $>65$ years-old while Kim et al. (2014) recruited adults aged 20 years and over [30].

One of limitations of the present study is the lack of longitudinal observations due to the cross-sectional nature of the study design. In addition, physical exercise was not objectively measured, and diet quality that may correlate with physical activity was not assessed. Furthermore, the participants with reduced eGFR are likely to be sicker and therefore are less likely to engage in exercise, so the possibility of reverse causality between reduced eGFR and physical activity engagement cannot not be ruled out. However, our findings based on a large cohort data extracted from the Taipei City Elderly Health Examination Database should represent the generalized elderly population. Further studies adopting a longitudinal design or prospective enrollment of elderly participants with objective physical exercise measurements may assist to confirm the results of the present study.

\section{Conclusions}

The present study found that physical exercise, among other favorable lifestyle habits, is beneficially associated with eGFR in a large cohort of community-dwelling elderly people aged $>65$ years. The reduced odds in having an eGFR $<60$ was significantly associated with occasional or regular physical in the presence or absence of CVD, hypertension, obesity, and diabetes, which are known to increase the risk of CKD. Future studies utilizing either longitudinal observation, prospective design, or active measurement of physical exercise levels should confirm our current findings of kidney function in the elderly and assist strategic planning of relevant public health policy.

\section{Abbreviations}

aOR: Adjusted odds ratio; BMl: Body mass index; BUN: Blood urea nitrogen; CKD: Chronic kidney disease; CKD-EPI: Chronic Kidney Disease Epidemiology Collaboration; CVD: Cardiovascular disease; eGFR: Estimated Glomerular filtration rate; HDL: High-density lipoprotein; KNHANES: Korean National Health and Nutrition Examination Survey; SD: Standard deviation

\section{Acknowledgements}

We would like to appreciate the participants of the Taipei City Elderly Health Examination Database and the staff numbers of the Taipei City Hospital for their contribution of the data and data collection.

\section{Authors' contributions}

All authors made substantial contributions to the conception and design, analysis and interpretation of data, and guarantor of integrity of the entire study. HJC statistical analysis, experimental studies, administrative, technical or material support, critical revision of the manuscript, final approval of the manuscript; KRL literature research, experimental studies, acquisition of data, statistical analysis, drafting of the manuscript, final approval of the manuscript; MTL literature research, clinical studies, drafting of the manuscript, definition of intellectual content; JLC definition of intellectual content, clinical studies, administrative, technical or material support critical revision of the manuscript. The author(s) read and approved the final manuscript.

Funding

None.

\section{Availability of data and materials}

The data analyzed for the current study are available from the corresponding author on reasonable request.

\section{Ethics approval and consent to participate}

No ethics committee approval was required. All participants signed a written informed consent at the time of health examination, authorizing the Taipei City Government access to their data for research purposes.

\section{Consent for publication}

Not applicable.

\section{Competing interests}

The authors declare that they have no competing interests.

\section{Author details}

${ }^{1}$ Department of Management Sciences, Tamkang University, No.151, Yingzhuan Rd., Tamsui Dist, New Taipei City 25137, Taiwan, Republic of China. ${ }^{2}$ Department of Pathology \& Laboratory Medicine, Taoyuan Armed Forces General Hospital, No.168, Chung-Shing Rd., Long-Tang District, Taoyuan City 325, Taiwan, Republic of China. ${ }^{3}$ Biomedical Engineering Department, Ming Chuan University, Taoyuan City 333, Taiwan.

Received: 13 August 2019 Accepted: 3 May 2020

Published online: 08 May 2020

\section{References}

1. Hill NR, Fatoba ST, Oke JL, Hirst JA, O'Callaghan CA, Lasserson DS, et al. Global prevalence of chronic kidney disease - a systematic review and meta-analysis. PLoS One. 2016;11(7):e0158765.

2. Hwang SJ, Tsai JC, Chen HC. Epidemiology, impact and preventive care of chronic kidney disease in Taiwan. Nephrology (Carlton). 2010; 15(Suppl 2):3-9.

3. Shin HY, Kang HT. Recent trends in the prevalence of chronic kidney disease in Korean adults: Korean National Health and nutrition examination survey from 1998 to 2013. J Nephrol. 2016;29(6):799-807.

4. Bruck K, Stel VS, Gambaro G, Hallan S, Volzke H, Arnlov J, et al. CKD prevalence varies across the European general population. J Am Soc Nephrol. 2016;27(7):2135-47.

5. Coresh J, Selvin E, Stevens LA, Manzi J, Kusek JW, Eggers P, et al. Prevalence of chronic kidney disease in the United States. JAMA. 2007;298(17):2038-47. 
6. Kidney Disease Improving Global Outcomes CKD Work Group: KDIGO 2012 clinical practice guideline for the evaluation and management of chronic kidney disease. Kidney Int. 2013;Supplement (3):1-150.

7. Hallan S, Astor B, Romundstad S, Aasarod K, Kvenild K, Coresh J. Association of kidney function and albuminuria with cardiovascular mortality in older vs younger individuals: the HUNT II study. Arch Intern Med. 2007;167(22):2490-6.

8. Manjunath G, Tighiouart H, Coresh J, Macleod B, Salem DN, Griffith JL, et al. Level of kidney function as a risk factor for cardiovascular outcomes in the elderly. Kidney Int. 2003;63(3):1121-9.

9. Tonelli M, Wiebe N, Culleton B, House A, Rabbat C, Fok M, et al. Chronic kidney disease and mortality risk: a systematic review. J Am Soc Nephrol. 2006;17(7):2034-47.

10. Hallan SI, Matsushita K, Sang Y, Mahmoodi BK, Black C, Ishani A, et al. Age and association of kidney measures with mortality and end-stage renal disease. JAMA. 2012;308(22):2349-60.

11. Liu C, Chen H, Liu C, Fu C, Zhang H, Yang H, et al. Combined application of eGFR and albuminuria for the precise diagnosis of stage 2 and $3 a$ CKD in the elderly. J Nephrol. 2014;27(3):289-97.

12. Kazancioglu R. Risk factors for chronic kidney disease: an update. Kidney Int Suppl (2011). 2013;3(4):368-71.

13. Ou SM, Chen YT, Shih CJ, Tarng DC. Impact of physical activity on the association between lipid profiles and mortality among older people. Sci Rep. 2017;7(1):8399.

14. de Labra C, Guimaraes-Pinheiro C, Maseda A, Lorenzo T, Millan-Calenti JC. Effects of physical exercise interventions in frail older adults: a systematic review of randomized controlled trials. BMC Geriatr. 2015;15:154.

15. Lavie CJ, Thomas RJ, Squires RW, Allison TG, Milani RV. Exercise training and cardiac rehabilitation in primary and secondary prevention of coronary heart disease. Mayo Clin Proc. 2009;84(4):373-83.

16. Roshanravan B, Robinson-Cohen C, Patel KV, Ayers E, Littman AJ, de Boer $\mathbb{H}$, et al. Association between physical performance and all-cause mortality in CKD. J Am Soc Nephrol. 2013;24(5):822-30.

17. Parsons TJ, Sartini C, Ash S, Lennon LT, Wannamethee SG, Lee IM, et al. Objectively measured physical activity and kidney function in older men; a cross-sectional population-based study. Age Ageing. 2017;46(6):1010-4.

18. Foster MC, Hwang SJ, Massaro JM, Jacques PF, Fox CS, Chu AY. Lifestyle factors and indices of kidney function in the Framingham heart study. Am J Nephrol. 2015;41(4-5):267-74.

19. He W, Goodkind D, Kowal P. bureau USC, editor. An Aging World: 2015, vol. 2016. Washington, DC: Government Publishing Office. p. P95/16-1.

20. Stevens LA, Viswanathan G, Weiner DE. Chronic kidney disease and endstage renal disease in the elderly population: current prevalence, future projections, and clinical significance. Adv Chronic Kidney Dis. 2010;17(4): 293-301.

21. Chen YT, Shih CJ, Ou SM, Hung SC, Lin CH, Tarng DC, et al. Periodontal disease and risks of kidney function decline and mortality in older people: a community-based cohort study. Am J Kidney Dis. 2015;66(2):223-30.

22. Tseng WC, Chen YT, Lin YP, Ou SM, Yang CY, Lin CH, et al. Hyperuricemia predicts an early decline in renal function among older people: a community-based cohort study. Sci Rep. 2019;9(1):980.

23. Tseng TJ, Wu YS, Tang JH, Chiu YH, Lee YT, Fan IC, et al. Association between health behaviors and mood disorders among the elderly: a community-based cohort study. BMC Geriatr. 2019;19(1):60.

24. Matsushita K, Mahmoodi BK, Woodward M, Emberson JR, Jafar TH, Jee SH, et al. Comparison of risk prediction using the CKD-EPI equation and the MDRD study equation for estimated glomerular filtration rate. JAMA. 2012; 307(18):1941-51.

25. Martens RJH, van der Berg JD, Stehouwer CDA, Henry RMA, Bosma H, Dagnelie PC, et al. Amount and pattern of physical activity and sedentary behavior are associated with kidney function and kidney damage: the Maastricht study. PLoS One. 2018;13(4):e0195306.

26. Qiu Z, Zheng K, Zhang H, Feng J, Wang L, Zhou H. Physical exercise and patients with chronic renal failure: a meta-analysis. Biomed Res Int. 2017; 2017:7191826

27. Greenwood SA, Koufaki P, Mercer TH, MacLaughlin HL, Rush R, Lindup H, et al. Effect of exercise training on estimated GFR, vascular health, and cardiorespiratory fitness in patients with CKD: a pilot randomized controlled trial. Am J Kidney Dis. 2015;65(3):425-34.
28. Kang IM, Chou CY, Tseng YH, Huang CC, Ho WY, Shih CM, et al. Association between betelnut chewing and chronic kidney disease in adults. J Occup Environ Med. 2007:49(7):776-9.

29. Roehm B, Simoni J, Pruszynski J, Wesson DE. Cigarette smoking attenuates kidney protection by angiotensin-converting enzyme inhibition in nondiabetic chronic kidney disease. Am J Nephrol. 2017;46(4):260-7.

30. Kim HN, Kim SH, Song SW. Is alcohol drinking associated with renal impairment in the general population of South Korea? Kidney Blood Press Res. 2014;39(1):40-9.

\section{Publisher's Note}

Springer Nature remains neutral with regard to jurisdictional claims in published maps and institutional affiliations.
Ready to submit your research? Choose BMC and benefit from:

- fast, convenient online submission

- thorough peer review by experienced researchers in your field

- rapid publication on acceptance

- support for research data, including large and complex data types

- gold Open Access which fosters wider collaboration and increased citations

- maximum visibility for your research: over $100 \mathrm{M}$ website views per year

At $\mathrm{BMC}$, research is always in progress.

Learn more biomedcentral.com/submissions 